\title{
Heterose em híbridos de melancia
}

\section{Aline Torquato Tavares ${ }^{1}$, Danilo Alves Porto da Silva Lopes ${ }^{1}$, Francielly Quiteria Guimaraes Alves $^{1}$, Gil Rodrigues Santos ${ }^{1}$, Ildon Rodrigues Nascimento ${ }^{1}$}

\footnotetext{
${ }^{1}$ Universidade Federal do Tocantins, Campus Universitário de Gurupi, Gurupi, Tocantins, Brasil. E-mail: alinet4t@ hotmail.com,
} danillo_sanfa@outlook.com, franciellyquiteria@yahoo.com.br, gilrsan@uft.edu.br, ildon@mail.uft.edu.br

Recebido: 30/04/2018; Aceito: 14/12/2018.

\section{RESUMO}

A melancia é uma curcubitácea bastante difundida no mundo, predominando nos cultivos comerciais o uso de híbridos. Apesar desse cenário, poucos são os trabalhos que relatam a magnitude e efeito da heterose em caracteres de interesse nesta cultura. O objetivo desse trabalho foi estimar a heterose em híbridos de melancia para características agronômicas e qualidade dos frutos. Os tratamentos foram constituídos de 22 genótipos, sendo 15 híbridos experimentais obtidos do cruzamento de seis genitores: quatro cultivares comerciais, duas linhagens experimentais e um híbrido comercial, usado como testemunha. O experimento foi realizado em delineamento de blocos casualizados com três repetições. Os cruzamentos e a análise estatística dos dados foram realizados segundo o modelo Gardner e Eberhart (1966) e adaptados por Miranda Filho e Geraldi (1984). Para heterose padrão, o híbrido Crimson Sweet ${ }^{\circledR}$ (Tecnoseed) x WMX-001G-14-02-55-01pl\#09 manifestou heterose positiva para a maioria das características. Para teores de açúcares, o maior percentual de heterose positiva foi encontrado no híbrido Crimson Sweet ${ }^{\circledR}$ (Hollar) x WMX-001G-14-02-55-01pl\#08. A melhor combinação híbrida para produtividade e firmeza da casca foi obtida nos cruzamentos com as linhagens experimentais WMX-001G-14-0255-01pl\#08 e WMX-001G-14-02-55-01pl\#09.

Palavras-chave: Citrullus lanatus L., dialelo, hibridação.

\section{Heterosis in watermelon hybrids}

\begin{abstract}
The watermelon is a widespread Cucurbitaceae in the world, predominating in commercial crops the use of hybrids. Despite this scenario, few studies report the magnitude and effect of heterosis on characters of interest in watermelon. This study aimed to estimate heterosis in hybrids of watermelon through diallel cross for agronomic traits associated with fruit quality. The treatments consisted of 22 genotypes, 15 experimental hybrids produced by breeding of six parents: four commercial cultivars, two experimental lines, and a commercial hybrid, used as the control. The experiment was conducted at the Federal University of Tocantins, Campus Gurupi, in a design of randomized block with three replications. The crosses and the statistical analysis of the data were performed according to the model Gardner and Eberhart (1966) and adapted by Miranda Filho and Geraldi (1984). For standard heterosis, the Crimson Sweet ${ }^{\circledR}$ (Tecnoseed) x WMX-001G-14-02-55-01pl\#09 hybrid manifested positive heterosis for most characteristics. For contents of sugars the highest percentage of positive heterosis was found in the hybrid Crimson Sweet ${ }^{\circledast}$ (Hollar) x WMX-001G-14-02-55-01pl\#08. The best hybrid combination for productivity and firmness of the shell was obtained in crosses with the experimental lines WMX-001G-14-02-5501pl\#08 and WMX-001G -14-02-55-01pl\#09.
\end{abstract}

Keywords: Citrullus lanatus L., diallel, hybridization. 


\section{Introdução}

A melancia (Citrullus lanatus (Thunb.) (Matsum e Nakai) pertence à família Cucurbitácea e tem como centro de origem primária o continente africano. É bastante apreciada nas regiões tropicais, sendo considerada uma das hortaliças mais importantes no Brasil (Oliveira et al., 2015).

O cultivo de melancia no estado do Tocantins, considerado o segundo maior produtor do país, tem predominado em regiões de várzeas, onde se pratica a irrigação por subsuperfície nas cidades de Lagoa da Confusão e Formoso do Araguaia, consideradas as principais produtoras desta olerícola no estado (Seagro, 2017). No Brasil, os principais cultivares utilizados pelos produtores caracterizam-se pela elevada produtividade de frutos de formato redondo e coloração vermelha intensa da polpa, considerada um dos atributos mais importantes quanto à sua aceitação no mercado (Embrapa, 2009).

Quando o objetivo é obter sementes através de cruzamentos específicos entre materiais genéticos distintos ou autofecundação de um mesmo material, para melancia é necessário realizar polinizações manuais e controladas, isto, por ser uma planta alógama. Casos assim são comuns em programas de melhoramento genético, principalmente em produção comercial de híbridos (Bomfim et al., 2013). No melhoramento de espécies olerícolas ou frutíferas, o aspecto visual e o tamanho do fruto são, muitas vezes, mais importantes do que a própria produtividade (Borém e Miranda, 2009).

Quando o interesse do melhorista refere-se ao desenvolvimento de híbrido, o estudo da heterose é fundamental para escolha das combinações mais promissoras (Souza et al., 2013), visto que os cultivares híbridos são mais vigorosos e promissores, uniformes e resistentes comparados aos não híbridos (Barros et al., 2010). O híbrido expressa heterose quando é superior à média dos genitores, podendo ser considerada heterose positiva quando uma característica avaliada em um híbrido é maior, ou heterose negativa quando a característica é menor (Borém e Miranda, 2013).

A heterose é um termo utilizado para descrever a superioridade de uma combinação híbrida em relação à média dos seus genitores (Bos e Caligari, 2011). As hipóteses que explicam a heterose são as de dominância, que atribui à presença de dominância parcial ou completa, e a de sobredominância, que atribui ao fato de o valor heterozigótico ser maior do que o valor do homozigótico (Fehr, 1987).

Para que então ocorra heterose, é necessário que exista dominância, pois locos sem dominância não geram heterose. Se alguns forem dominantes em direções contrárias, seus efeitos tenderão a se cancelar e nenhuma heterose poderá ser observada, apesar da dominância nos locos individuais (Falconer, 1987). Ainda não foi possível provar sequer uma das hipóteses que explica o fenômeno da heterose, pela complexidade da herança das características quantitativas; é possível que as duas hipóteses se complementem para uma explicação completa da heterose (Borém e Miranda, 2009).

Uma ferramenta que auxilia na escolha de progenitores com base em novos valores genéticos e, principalmente, considerando a sua capacidade de combinação em híbridos que produzam populações segregantes promissoras, é a de cruzamento dialélico. Nesse sentido, os cruzamentos dialélicos podem auxiliar na escolha dos melhores híbridos disponíveis no mercado para geração de populações ou para exploração na forma de híbridos (Pfann et al., 2009). Objetivou-se, com este trabalho, estimar a heterose em híbridos de melancia a partir de cruzamento dialélico.

\section{Material e Métodos}

$\mathrm{O}$ experimento foi conduzido na área experimental da Universidade Federal do Tocantins - UFT, Campus Universitário de Gurupi, no ano agrícola 2011/2012. O material genético foi constituído de 22 genótipos, sendo 15 híbridos experimentais obtidos do cruzamento de seis genitores, que foram: quatro cultivares comercias [Sandia ${ }^{\circledR}$ (Feltrin); Crimson Sweet ${ }^{\circledR}$ (Hollar); Crimson Sweet $^{\circledR}$ (Tecnoseed); Crimson Sweet ${ }^{\circledR}$ (Topseed); duas linhagens experimentais (WMX-001G-14-02-5501pl\#08 e WMX-001G-14-02-55-01pl\#09)] e um híbrido comercial, Top Gun ${ }^{\circledR}$ (Syngenta), usado como testemunha.

As linhagens experimentais WMX-001G-14-02-5501pl\#08 e WMX-001G-14-02-55-01pl\#09 foram obtidas do cruzamento controlado do acesso africano PI 595201 com um cultivar comercial de frutos do tipo Crimson Sweet, avançado por retrocruzamento e selecionado para tolerância genética a isolados locais de PRSV - W (Pappaya rings pot vírus).

Os híbridos experimentais foram obtidos de cruzamentos manuais e controlados entre os genótipos em campo na estação experimental da UFT, Campus de Gurupi, TO. Para o procedimento, grupos de cinco plantas de cada genitor (feminino ou masculino) foram transplantados (as mudas foram obtidas em bandejas de poliestireno expandido com 72 células) para campo no estádio de quatro folhas definitivas. Os genitores masculinos foram transplantados com 10 dias de antecedência para garantir a produção de pólen.

Para realizar os cruzamentos, os botões florais femininos que abririam no dia seguinte foram selecionados e identificados no final de cada dia, e, em seguida, protegidos conforme Ferreira (2005). As polinizações foram realizadas pela manhã, período de 
maior visitação das abelhas. Procedeu-se, nesse mesmo dia, à retirada da flor masculina aberta de cada genitor masculino, fazendo-se a polinização das flores femininas abertas em cada genitor (Ferreira, 2005). Cada cruzamento foi identificado com um fio de lã de cores variadas, conforme o genitor masculino utilizado, em que: 1 - amarelo; 2 - azul; 3 - branco; 4 - cinza; 5 preto e 6 - vermelho, que representou cada genitor. As sementes dos cruzamentos foram obtidas em pelo menos dois frutos completamente desenvolvidos e maduros fisiologicamente.

As sementes foram retiradas dos frutos, lavadas, secas à sombra, colocadas em sacos de papel, identificadas e armazenadas em local com temperatura e umidade controladas. Os 20 tratamentos foram avaliados em condições de campo, utilizando-se o delineamento experimental em blocos casualizados com três repetições. A parcela experimental foi constituída de uma única linha com seis plantas, com o espaçamento de 2,65 x 0,65 m entre linhas e entre plantas dentro das linhas, respectivamente, tendo a colheita iniciado 75 dias após o plantio. O preparo do solo e adubação de plantio foram realizados segundo critérios de adubação utilizados pelos produtores da região, com $200 \mathrm{~kg} \mathrm{ha}{ }^{-1}$ de $\mathrm{P}_{2} \mathrm{O}_{5}$ na forma de superfosfato simples, $100 \mathrm{~kg} \mathrm{ha}^{-1} \mathrm{de}_{2} \mathrm{O}$ na forma de cloreto de potássio e $50 \mathrm{~kg} \mathrm{ha}^{-1}$ de nitrogênio na forma de uréia. Para não prejudicar a germinação, apenas metade da dose de $\mathrm{K}_{2} \mathrm{O}$ foi colocada na linha de plantio, sendo a outra metade distribuída ao lado da mesma. Aos 15 dias após a germinação, foi realizada adubação de cobertura com $100 \mathrm{~kg} \mathrm{ha}^{-1}$ na forma de 20-00-20. Os tratos culturais foram realizados de acordo com recomendação para a cultura.

As características avaliadas foram: produtividade total de frutos (PROD em t. ha ${ }^{-1}$ ); massa média de frutos em kg fruto $^{-1}$ (MMF); formato do fruto (FORM) conforme Silva et al. (2006); espessura da casca em milímetros (EC); sólidos solúveis ( $\left.{ }^{\circ} \mathrm{BRIX}\right)$; firmeza da casca em Newton (FC); firmeza da polpa em Newton (FP). Avaliou-se também a coloração da polpa (CP) conforme a escala de notas, sendo: 1 - vermelha, 2 rosa-intenso, 3 - rosa-médio, 4 - rosa-claro e 5 - branca; e coloração externa do fruto (CEF) conforme escala de nota, em que: 1 - verde-escuro, 2 - verde-médio, 3 verde-claro e 4 -amarelo, sendo o padrão de listras da casca (PLC) obtido segundo escala de notas, conforme metodologia proposta por Silva et al. (2006), em que: 1 - sem listras, 2 - listra larga, 3 - listra estreita e 4 - mosqueado. Procedeu-se à análise de variância para cada um dos caracteres avaliados para o delineamento experimental do tipo blocos casualizados, conforme modelo estatístico: $Y_{i j}=m+b_{j}+t_{i}+e_{i j}$, em que: Yij: é o valor observado do tratamento i $(\mathrm{i}=1,2, \ldots, 81)$, no bloco $\mathrm{j}(\mathrm{j}=1,2,3)$; $\mathrm{m}$ : é a constante inerente a todas as observações; bj: é o efeito do bloco j; ti: é o efeito do tratamento i; eij: é o erro experimental aleatório associado à observação Yij $\mathrm{N}\left(0, \mathrm{~S}^{2} \mathrm{e}\right)$. As médias dos genótipos foram agrupadas pelo teste de Scott-Knott (1974) $(\mathrm{p} \geq 0,05)$.

O modelo da análise de variância e os graus de liberdade entre os tratamentos do dialelo foram desdobrados segundo o modelo proposto por Gardner e Eberhart (1966) e adaptado por Miranda Filho e Geraldi (1984). A heterose tradicional foi calculada pela diferença entre o valor do híbrido $\left(\mathrm{F}_{1}\right)$ e a média de seus pais $\left(\mathrm{P}_{1}\right.$ e $\left.\mathrm{P}_{2}\right)$.

$$
H T(\%)=\frac{\bar{F}_{1-}\left(\bar{P}_{1}+\bar{P}_{2} / 2\right)}{\left(\bar{P}_{1}+\bar{P}_{2} / 2\right)} \times 100
$$

em que $H T(\%)$ é a heterose tradicional em porcentagem; $\bar{F}_{1}$ a média do cruzamento entre os genitores $\mathrm{P}_{1}$ e $\mathrm{P}_{2}$; e $\bar{P}_{1}$ e $\bar{P}_{2}$ a média dos genitores 1 e 2. A Heterose Padrão foi estimada em relação a um cultivar padrão $(\mathrm{CP})$.

$$
H P(\%)=\frac{\bar{F}_{1}-\overline{C P}}{\overline{C P}} x 100
$$

em que $H P(\%)$ é a heterose em porcentagem; $\bar{F}_{1}$ a média do cruzamento entre os genitores $\mathrm{P}_{1}$ e $\mathrm{P}_{2}$; e $\overline{C P}$ a média do cultivar padrão (Híbrido Top Gun). Os dados médios de cada tratamento para cada característica avaliada foram submetidos à análise de variância, com seus devidos desdobramentos por meio do programa Genes (Cruz, 2006).

\section{Resultados e Discussão}

Para as médias das características avaliadas (Tabela 1), houve diferença significativa entre o cultivar padrão Top Gun e os híbridos avaliados; a produtividade média de frutos variou entre os genótipos, de 18,7 $\mathrm{t} \mathrm{ha}^{-1}$ (WMX-001G-14-02-55-01pl\#08) a 36,7 $\mathrm{t} \mathrm{ha}^{-1}$ (Sandia ${ }^{\circledR}$ (Feltrin); entre os cruzamentos, a maior média encontrada foi para Crimson Sweet ${ }^{\circledR}$ (Hollar) x WMX001G-14-02-55-01pl\#08, com 29,1 t ha ${ }^{-1}$.

Em geral, a massa média dos frutos colhidos (Tabela 1) foi superior ao mínimo exigido pelo mercado interno, que, segundo Martins et al. (2013), deve ser superior a 7 $\mathrm{kg}$ para obter maior aceitação comercial, sendo a melhor combinação híbrida Crimson Sweet ${ }^{\circledR}$ (Hollar) x WMX001G-14-02-55-01pl\#08, com peso médio de frutos igual a $8,5 \mathrm{~kg}$ fruto $^{-1}$. 
Tabela 1. Médias para produtividade em $\mathrm{t} \mathrm{ha} \mathrm{h}^{-1}$ (PROD), massa média de frutos em $\mathrm{kg}_{\text {fruto }}{ }^{-1}$ (MMF), formato do fruto (FF), espessura da casca em mm (EC), teores de açúcares ( $\left.{ }^{\circ} \mathrm{BRIX}\right)$, firmeza da casca em $\mathrm{N}$ (FC), firmeza da polpa em $\mathrm{N}$ (FP), coloração da polpa (CP), coloração externa do fruto (CEF) e padrão de listras da casca (PLC) em frutos de híbridos de melancia.

\begin{tabular}{ccccccccccc}
\hline Genótipo & PROD & MMF & FF & EC & BRIX & FC & FP & CP & CEF & PLC \\
\hline 1 & $36,7 \mathrm{a}$ & $9,8 \mathrm{~b}$ & $1,1 \mathrm{~b}$ & $19,5 \mathrm{c}$ & $10,9 \mathrm{~b}$ & $52,0 \mathrm{a}$ & $4,4 \mathrm{c}$ & $2,1 \mathrm{c}$ & $0,7 \mathrm{c}$ & $4,2 \mathrm{a}$ \\
2 & $31,2 \mathrm{a}$ & $8,1 \mathrm{c}$ & $1,0 \mathrm{c}$ & $20,4 \mathrm{c}$ & $12,0 \mathrm{a}$ & $44,8 \mathrm{a}$ & $8,5 \mathrm{c}$ & $1,8 \mathrm{c}$ & $1,2 \mathrm{c}$ & $3,7 \mathrm{a}$ \\
3 & $28,3 \mathrm{~b}$ & $8,3 \mathrm{c}$ & $1,1 \mathrm{~b}$ & $20,4 \mathrm{c}$ & $10,4 \mathrm{~b}$ & $35,2 \mathrm{~b}$ & $4,7 \mathrm{c}$ & $1,8 \mathrm{c}$ & $2,0 \mathrm{~b}$ & $3,0 \mathrm{~b}$ \\
4 & $25,6 \mathrm{~b}$ & $9,2 \mathrm{~b}$ & $1,1 \mathrm{~b}$ & $22,8 \mathrm{a}$ & $11,1 \mathrm{~b}$ & $34,9 \mathrm{~b}$ & $4,6 \mathrm{c}$ & $2,3 \mathrm{c}$ & $1,7 \mathrm{~b}$ & $3,0 \mathrm{~b}$ \\
5 & $18,7 \mathrm{c}$ & $7,6 \mathrm{c}$ & $1,0 \mathrm{~b}$ & $21,0 \mathrm{~b}$ & $9,0 \mathrm{c}$ & $31,2 \mathrm{~b}$ & $16,4 \mathrm{a}$ & $2,3 \mathrm{c}$ & $1,6 \mathrm{~b}$ & $2,3 \mathrm{c}$ \\
6 & $20,7 \mathrm{c}$ & $6,4 \mathrm{c}$ & $1,0 \mathrm{c}$ & $19,0 \mathrm{c}$ & $11,3 \mathrm{~b}$ & $31,7 \mathrm{~b}$ & $7,2 \mathrm{c}$ & $2,7 \mathrm{c}$ & $2,0 \mathrm{~b}$ & $3,0 \mathrm{~b}$ \\
$1 \mathrm{x} 2$ & $21,3 \mathrm{c}$ & $8,2 \mathrm{c}$ & $1,1 \mathrm{~b}$ & $20,9 \mathrm{~b}$ & $8,7 \mathrm{c}$ & $36,5 \mathrm{~b}$ & $11,3 \mathrm{~b}$ & $4,2 \mathrm{a}$ & $2,6 \mathrm{a}$ & $2,6 \mathrm{~b}$ \\
$1 \times 3$ & $26,7 \mathrm{~b}$ & $6,0 \mathrm{c}$ & $1,2 \mathrm{a}$ & $18,5 \mathrm{c}$ & $8,1 \mathrm{c}$ & $36,2 \mathrm{~b}$ & $15,8 \mathrm{a}$ & $4,5 \mathrm{a}$ & $2,6 \mathrm{a}$ & $1,6 \mathrm{c}$ \\
$1 \times 4$ & - & - & - & - & - & - & - & - & - & - \\
$1 \times 5$ & $25,2 \mathrm{~b}$ & $7,5 \mathrm{c}$ & $1,1 \mathrm{~b}$ & $20,0 \mathrm{c}$ & $11,6 \mathrm{a}$ & $30,9 \mathrm{~b}$ & $7,3 \mathrm{c}$ & $2,6 \mathrm{c}$ & $2,6 \mathrm{a}$ & $3,0 \mathrm{~b}$ \\
$1 \times 6$ & $24,3 \mathrm{~b}$ & $7,3 \mathrm{c}$ & $1,1 \mathrm{~b}$ & $18,0 \mathrm{c}$ & $11,5 \mathrm{~b}$ & $32,2 \mathrm{~b}$ & $7,1 \mathrm{c}$ & $2,0 \mathrm{c}$ & $2,0 \mathrm{~b}$ & $3,0 \mathrm{~b}$ \\
$2 \times 3$ & - & - & - & - & - & - & - & - & - & - \\
$2 \times 4$ & $25,8 \mathrm{~b}$ & $7,2 \mathrm{c}$ & $1,1 \mathrm{~b}$ & $22,0 \mathrm{~b}$ & $9,9 \mathrm{c}$ & $36,3 \mathrm{~b}$ & $9,1 \mathrm{c}$ & $3,3 \mathrm{~b}$ & $2,6 \mathrm{a}$ & $3,0 \mathrm{~b}$ \\
$2 \times 5$ & $29,1 \mathrm{~b}$ & $8,5 \mathrm{c}$ & $1,1 \mathrm{~b}$ & $19,3 \mathrm{c}$ & $12,6 \mathrm{a}$ & $35,1 \mathrm{~b}$ & $7,4 \mathrm{c}$ & $2,3 \mathrm{c}$ & $2,0 \mathrm{~b}$ & $3,0 \mathrm{~b}$ \\
$2 \times 6$ & $26,4 \mathrm{~b}$ & $7,8 \mathrm{c}$ & $1,1 \mathrm{~b}$ & $18,6 \mathrm{c}$ & $8,7 \mathrm{c}$ & $32,7 \mathrm{~b}$ & $8,2 \mathrm{c}$ & $3,3 \mathrm{~b}$ & $1,6 \mathrm{~b}$ & $3,0 \mathrm{~b}$ \\
$3 \times 4$ & $27,9 \mathrm{~b}$ & $8,2 \mathrm{c}$ & $1,1 \mathrm{~b}$ & $19,3 \mathrm{c}$ & $9,6 \mathrm{c}$ & $34,5 \mathrm{~b}$ & $12,4 \mathrm{~b}$ & $3,3 \mathrm{~b}$ & $3,0 \mathrm{a}$ & $2,0 \mathrm{c}$ \\
$3 \times 5$ & $22,2 \mathrm{c}$ & $6,8 \mathrm{c}$ & $1,1 \mathrm{~b}$ & $17,8 \mathrm{c}$ & $8,0 \mathrm{c}$ & $36,9 \mathrm{~b}$ & $13,3 \mathrm{~b}$ & $4,8 \mathrm{a}$ & $3,0 \mathrm{a}$ & $2,0 \mathrm{c}$ \\
$3 \times 6$ & $27,2 \mathrm{~b}$ & $8,0 \mathrm{c}$ & $1,1 \mathrm{~b}$ & $20,2 \mathrm{c}$ & $11,9 \mathrm{a}$ & $35,6 \mathrm{~b}$ & $5,5 \mathrm{c}$ & $2,4 \mathrm{c}$ & $1,7 \mathrm{~b}$ & $3,2 \mathrm{~b}$ \\
$4 \times 5$ & $21,8 \mathrm{c}$ & $6,7 \mathrm{c}$ & $1,0 \mathrm{c}$ & $23,0 \mathrm{a}$ & $10,5 \mathrm{~b}$ & $25,7 \mathrm{c}$ & $8,6 \mathrm{c}$ & $3,0 \mathrm{c}$ & $2,0 \mathrm{~b}$ & $3,0 \mathrm{~b}$ \\
$4 \times 6$ & $20,6 \mathrm{c}$ & $6,4 \mathrm{c}$ & $1,1 \mathrm{~b}$ & $20,0 \mathrm{c}$ & $9,8 \mathrm{c}$ & $29,0 \mathrm{c}$ & $6,2 \mathrm{c}$ & $1,0 \mathrm{c}$ & $2,0 \mathrm{~b}$ & $3,0 \mathrm{~b}$ \\
$5 \times 6$ & $24,2 \mathrm{~b}$ & $8,1 \mathrm{c}$ & $1,0 \mathrm{c}$ & $23,0 \mathrm{a}$ & $8,0 \mathrm{c}$ & $24,4 \mathrm{c}$ & $14,9 \mathrm{a}$ & $4,0 \mathrm{a}$ & $2,0 \mathrm{~b}$ & $3,0 \mathrm{~b}$ \\
Top Gun & $36,5 \mathrm{a}$ & $11,5 \mathrm{a}$ & $1,0 \mathrm{c}$ & $18,9 \mathrm{c}$ & $11,6 \mathrm{a}$ & $25,7 \mathrm{c}$ & $6,2 \mathrm{c}$ & $2,4 \mathrm{c}$ & $3,2 \mathrm{a}$ & $3,0 \mathrm{~b}$ \\
\hline
\end{tabular}

Médias seguidas de mesma letra não diferem pelo teste de Scott-Knott (1974) (p $\geq 0,05)$. 1- Sandia ${ }^{\circledR}$ (Feltrin); 2 - Crimson Sweet ${ }^{\circledR}$ (Hollar); 3 Crimson Sweet ${ }^{\circledR}$ (Tecnoseed); 4 - Crimson Sweet ${ }^{\circledR}$ (Topseed); 5 - WMX-001G-14-02-55-01pl\#08 e 6 - WMX-001G-14-02-55-01pl\#09.

Em relação ao formato de fruto, todos apresentaram valores iguais ou bem próximos a 1,0, mantendo um padrão adequado entre os genitores e cruzamentos analisados (Tabela 1). A espessura da casca variou de $17,8 \mathrm{~mm}$ no cruzamento Crimson Sweet ${ }^{\circledR}$ (Tecnoseed) x WMX-001G-14-02-55-01pl\#08 a $23 \mathrm{~mm}$ em duas das combinações (Crimson Sweet ${ }^{\circledR}$ (Topseed) x WMX001G-14-02-55-01pl\#08 e WMX-001G-14-02-5501pl\#08 x WMX-001G-14-02-55-01pl\#09), espessuras essas que estão dentro dos grupos 1 e 2 propostos por Gusmini e Wehner (2004), $1^{\circ}$ grupo $>19 \mathrm{~mm}, 2^{\circ}$ grupo $10 \mathrm{a} 19 \mathrm{~mm}$.

A combinação híbrida com maior valor de ${ }^{\circ} \mathrm{BRIX}$ foi Crimson Sweet $^{\circledR}$ (Hollar) x WMX-001G-14-02-55$01 \mathrm{pl} \# 08$, com $12,6 \%$ (Tabela 1 ), inclusive maior que no cultivar Top Gun. Essa característica é muito valorizada, visto que quanto maior o teor de sólidos solúveis totais, maior será a aceitação comercial desse fruto. Apesar dos genótipos também serem selecionados para que seus frutos apresentassem um maior teor de açúcar, a exploração na forma de híbridos tende a resultar em combinações superiores quando os dois genitores são selecionados para essa característica.

Quanto à firmeza da casca, o Sandia ${ }^{\circledR}$ (Feltrin) e o Crimson Sweet ${ }^{\circledR}$ (Hollar) apresentaram maiores valores (52 e 44,8 N), respectivamente, diferindo dos demais híbridos e cruzamentos, porém, dez desses cruzamentos não diferiram entre si estatisticamente, com valores entre 30,9 Sandia $^{\circledR}$ (Feltrin) x WMX-001G-14-02-5501pl\#08 e 36,9 N (Crimson Sweet ${ }^{\circledR}$ (Tecnoseed) x WMX-001G-14-02-55-01pl\#08), apresentando um padrão bem próximo dos outros quatro genitores.

Tratando-se da parte comestível do fruto de melancia, o consumidor busca um fruto com polpa de menor firmeza. Dentre os genótipos avaliados (Tabela 1), oito materiais não diferiram entre si, obtendo as menores médias para esse caractere, sendo a menor delas Crimson Sweet ${ }^{\circledR}$ (Topseed) x WMX-001G-14-02$55-01 \mathrm{pl} \# 09$, com 5,5 N.

Para característica coloração da polpa, quanto menor a nota, mais intensa é a coloração, portanto, o híbrido Crimson Sweet $^{\circledR}$ (Topseed) x WMX-001G-14-02-5501pl\#09 destacou-se entre os demais (nota 1,0), apresentando polpa vermelha intensa. Por outro lado, a combinação Crimson Sweet ${ }^{\circledR}$ (Tecnoseed) x WMX001G-14-02-55-01pl\#08 possui polpa quase branca (nota 4,8). Em média, a polpa dos híbridos variou de rosa-intenso (nota 2,0) a rosa (nota 4,0) (Tabela 1).

A cor externa do fruto é um caráter economicamente importante, uma vez que o consumidor prefere frutos padronizados do tipo "Crimson Sweet", cujos frutos são de cor externa verde clara; seis materiais apresentaram essa coloração, sendo assim uma característica que precisa ser melhorada. Essa característica também é influenciada pelo padrão de listra apresentado, onde dez cruzamentos apresentaram padrão de listras semelhante 
ao híbrido Top Gun ${ }^{\circledR}$ (Syngenta) (Tabela 1) e a três dos genitores.

A Tabela 2 refere-se aos efeitos de heterose tradicional para as características analisadas. Para produtividade, observou-se um efeito de heterose positiva para cinco dos cruzamentos, sendo WMX001G-14-02-55-01pl\#08 x WMX-001G-14-02-55$01 \mathrm{pl} \# 09$ o de maior valor $(22,8 \%)$, com média de $24,2 \mathrm{t}$ ha $^{-1}$ (Tabela 1). Godoy et al. (2008), em trabalho avaliando a produção e heterose em cruzamentos entre linhagens de pepino do grupo caipira, relataram que o híbrido mais produtivo não foi o de maior heterose.

Segundo os autores, valores de heterose elevados, por si só, não são sinônimos de alta produção, pois a heterose retrata superioridade (ou não) em relação à média dos genitores. Todavia, Marame et al. (2009) relatam que o uso dos híbridos é vantajoso, pois pode combinar caracteres importantes presentes em duas linhagens contrastantes; essa vantagem é ampliada pelo benefício da heterose em características importantes, como produtividade, qualidade e uniformidade. Os demais genótipos de heterose negativa demonstram decréscimo do vigor híbrido, destacando-se o Sandia ${ }^{\circledR}$ (Feltrin) x Crimson Sweet ${ }^{\circledR}$ (Hollar), com -37,3\% .

Como observado para a produtividade, a massa média de frutos também apresentou heterose positiva para os mesmos cruzamentos, exceto Crimson Sweet ${ }^{\circledR}$ (Tecnoseed) x Crimson Sweet ${ }^{\circledR}$ (Topseed), que obteve valor negativo (-6,3 t. ha- ${ }^{-1}$ (Tabela 2). De acordo com Leão et al. (2008), o aumento da produtividade não é influenciado exclusivamente pelo aumento no número de frutos, mas, também, pelo aumento da massa média destes, onde os autores constataram forte correlação entre a produtividade e a massa média de frutos.

Observou-se maior heterose positiva no híbrido proveniente dos genitores Sandia ${ }^{\circledR}$ (Feltrin) x Crimson Sweet ${ }^{\circledR}$ (Tecnoseed), com $9,9 \%$, e menor heterose negativa no híbrido Crimson Sweet $^{\circledR}$ (Topseed) $\mathrm{x}$ WMX-001G-14-02-55-01pl\#08, com-10,2\% t. ha ${ }^{-1} \mathrm{em}$ formato de fruto (Tabela 2).

Para espessura e firmeza da casca, o cruzamento que apresentou maiores valores de heterose positiva foi WMX-001G-14-02-55-01pl\#08 x WMX-001G-14-02$55-01 \mathrm{pl} \# 09$, com 15 e 78,4\%, respectivamente. Parentais cujos cruzamentos manifestam heterose alta e positiva apresentam divergência genética (Bernini e Paterniani, 2012).

A espessura de casca é uma característica importante para a resistência dos frutos ao manuseio e ao transporte, haja vista que, entre os cultivares utilizados no Brasil, aqueles de frutos com casca mais espessa são mais adequados ao tipo de transporte predominante no mercado interno: frutos a granel transportados em caminhões sem refrigeração, por longas distâncias (Souza et al., 2013). A espessura da casca maior garante maior resistência durante o transporte e maior durabilidade pós-colheita, além de diminuir os danos causados pelo ataque das brocas das cucurbitáceas (Diaphania spp.), que acarretam danos, impossibilitando a exportação da fruta para o mercado (Bahari et al., 2012).

No Brasil, a preferência do mercado consumidor leva em consideração o tamanho e o formato do fruto, a coloração da polpa e o teor de sólidos solúveis, considerados os caracteres de maior importância (Ramos et al., 2009); contudo, dentre as características mais expressivas, está o teor de açúcar. Foi observado um maior número de cruzamentos com heterose negativa se comparada às positivas, destacando o cruzamento Crimson Sweet ${ }^{\circledR}$ (Hollar) x WMX-001G14-02-55-01pl\#08, com 20\%.

De acordo com Martins et al. (2013), a firmeza da polpa é um atributo de qualidade importante, em razão dos frutos com maior firmeza serem mais resistentes às injúrias mecânicas durante $\mathrm{o}$ transporte $\mathrm{e} \quad \mathrm{a}$ comercialização. Os resultados para esta característica mostraram que a heterose em relação à média dos pais foi positiva para a maioria dos híbridos (Tabela 2), variando de $-40,6 \%$ para o híbrido Crimson Sweet $^{\circledR}$ (Hollar) x WMX-001G-14-02-55-01pl\#08 a 247,3\% para o híbrido Sandia ${ }^{\circledR}$ (Feltrin) x Crimson Sweet ${ }^{\circledR}$ (Tecnoseed).

A coloração da polpa para melancia é uma característica importante, pois o consumidor tornou-se mais exigente, optando por frutos com coloração da polpa vermelha intensa (Tavares et al., 2018). Houve efeito heterótico positivo para a maioria dos híbridos avaliados, sendo os maiores percentuais encontrados para Crimson Sweet ${ }^{\circledR}$ (Tecnoseed) x WMX-001G-1402-55-01pl\#08, Sandia ${ }^{\circledR}$ (Feltrin) x Crimson Sweet ${ }^{\circledR}$ (Tecnoseed) e Sandia ${ }^{\circledR}$ (Feltrin) x Crimson Sweet ${ }^{\circledR}$ (Hollar), com 134,1\%, 130,8\% e $115,4 \%$, respectivamente (Tabela 2). A coloração externa do fruto é outro fator determinante no momento da compra. Para essa característica, houve heterose positiva para onze dos híbridos avaliados (Tabela 2), demonstrando vigor híbrido em relação à média dos pais. Não foi observada heterose para combinação híbrida Crimson Sweet $^{\circledR}$ (Hollar) x WMX-001G-14-02-55-01pl\#09.

Para o padrão de listra da casca, não foi constatada heterose em dois híbridos $(2 \times 5$ e $4 \times 6)$, tendo maior desempenho positivo nos híbridos Crimson Sweet ${ }^{\circledR}$ (Topseed) x WMX-001G-14-02-55-01pl\#08 e WMX001G-14-02-55-01pl\#08 x WMX-001G-14-02-5501pl\#09, com 13,2\%.

Para a maioria das características avaliadas, o híbrido WMX-001G-14-02-55-01pl\#08 x WMX-001G14-02-55-01 pl\#09 apresentou heterose positiva. Segundo Cruz e Regazzi (1997), a maior heterose revelada pela combinação híbrida resulta em maior 
probabilidade de resgate de indivíduos superiores nas suas populações segregantes.

As estimativas percentuais dos efeitos de heterose padrão estão apresentadas na Tabela 3. As características produtividade e massa média dos frutos apresentaram heterose negativa para todos os híbridos avaliados, confirmando que existe simultaneidade entre estes dois caracteres para expressão da heterose. O mesmo foi observado para a característica coloração externa do fruto.

A endogamia acarreta perda de vigor em plantas alógamas, podendo ter ocorrido este fenômeno para produtividade e massa média de frutos. A perda de vigor em outras espécies vegetais está associada à presença de alelos deletérios e letais em genótipos homozigóticos.

Muitos genes recessivos permanecem ocultos em condições heterozigotas nas populações naturais, à medida que a homozigose aumenta nas populações endogâmicas, existe a probabilidade maior para que as características recessivas, muitas das quais são deletérias, comecem a se manifestar, resultando em perda de vigor.

Godoy et al. (2005) observaram, na cultura do pepino, que além de vigor pela endogamia, a redução na produção de frutos pode ter outra explicação: redução na frequência de plantas ginóicas com o aumento do número de autofecundações.

Para formato de frutos, firmeza da casca e da polpa (Tabela 3), verificou-se um predomínio de heterose positiva, estabelecendo ótima combinação entre estes cruzamentos para obtenção de vigor, com exceção dos cruzamentos (Crimson Sweet ${ }^{\circledR}$ (Topseed) x WMX001G-14-02-55-01pl\#08), (WMX-001G-14-02-5501pl\#08 x WMX-001G-14-02-55-01pl\#09) e (Crimson Sweet ${ }^{\circledR}$ (Tecnoseed) x WMX-001G-14-02-55-01pl\#09), respectivamente.

Tabela 2. Estimativas percentuais dos efeitos de heterose tradicional para as características produtividade em t. ha ${ }^{-1}$ (PROD), massa média de frutos em kg. fruto ${ }^{-1}$ (MMF), formato do fruto (FF), espessura da casca em mm (EC), teores de açúcares $\left({ }^{\circ} \mathrm{BRIX}\right)$, firmeza da casca em N (FC), firmeza da polpa em N (FP), coloração da polpa (CP), coloração externa do fruto (CEF) e padrão de listras da casca (PLC) em frutos de híbridos de melancia.

\begin{tabular}{crrrrrrrrrr}
\hline Híbridos & PROD & \multicolumn{1}{l}{ MMF } & \multicolumn{1}{l}{ FF } & \multicolumn{1}{l}{ EC } & \multicolumn{1}{l}{ BRIX } & \multicolumn{1}{l}{ FC } & \multicolumn{1}{l}{ FP } & CP & CEF & PLC \\
\hline $1 \times 2$ & $-37,3$ & $-8,4$ & 2,9 & 4,8 & $-24,0$ & $-24,6$ & 75,2 & 115,4 & 173,7 & $-34,2$ \\
$1 \times 3$ & $-17,8$ & $-31,5$ & 9,9 & $-7,3$ & $-23,9$ & $-17,0$ & 247,3 & 130,8 & 92,6 & $-55,6$ \\
$1 \times 5$ & $-9,0$ & $-13,8$ & $-0,5$ & $-1,2$ & 16,6 & $-25,7$ & $-29,8$ & 18,2 & 126,1 & $-7,7$ \\
$1 \times 6$ & $-15,3$ & $-9,9$ & 7,2 & $-6,5$ & 3,6 & $-23,1$ & 22,4 & $-16,7$ & 48,1 & $-16,7$ \\
$2 \times 4$ & $-9,2$ & $-16,8$ & 2,4 & 1,9 & $-14,3$ & $-8,9$ & 38,9 & 61,0 & 79,3 & $-10,4$ \\
$2 \times 5$ & 16,6 & 8,3 & 4,3 & $-6,8$ & 20,0 & $-7,6$ & $-40,6$ & 12,2 & 42,9 & 0,0 \\
$2 \times 6$ & 1,7 & 7,6 & 3,4 & $-5,6$ & $-25,3$ & $-14,5$ & 4,5 & 46,7 & 0,0 & $-10,4$ \\
$3 \times 4$ & 3,5 & $-6,3$ & 1,9 & $-10,6$ & $-10,7$ & $-1,6$ & 166,7 & 61,0 & 62,2 & $-33,3$ \\
$3 \times 5$ & $-5,5$ & $-14,5$ & 7,5 & $-14,0$ & $-17,5$ & 11,1 & 26,1 & 134,1 & 66,7 & $-24,5$ \\
$3 \times 6$ & 11,0 & 8,8 & 1,9 & 2,5 & 9,7 & 6,4 & $-7,6$ & 6,7 & $-15,0$ & 6,7 \\
$4 \times 5$ & $-1,6$ & $-20,2$ & $-10,2$ & 5,0 & 4,5 & $-22,2$ & $-18,1$ & 30,4 & 21,2 & 13,2 \\
$4 \times 6$ & $-11,0$ & $-17,9$ & 0,9 & $-4,3$ & $-12,5$ & $-12,9$ & 5,1 & $-60,0$ & 8,1 & 0,0 \\
$5 \times 6$ & 22,8 & 15,7 & $-1,0$ & 15,0 & $-21,2$ & 78,4 & 26,3 & 60,0 & 11,1 & 13,2 \\
\hline
\end{tabular}

1- Sandia $^{\circledR}$ (Feltrin); 2 - Crimson Sweet ${ }^{\circledR}$ (Hollar); 3 - Crimson Sweet ${ }^{\circledR}$ (Tecnoseed); 4 - Crimson Sweet ${ }^{\circledR}$ (Topseed); 5 - WMX-001G-14-02-5501pl\#08 e 6 - WMX-001G-14-02-55-01pl\#09.

Tabela 3. Estimativas percentuais dos efeitos de heterose padrão (\%) para as características produtividade em $\mathrm{t} \mathrm{ha}^{-1}$ (PROD), massa média de frutos em $\mathrm{kg}_{\text {fruto }}{ }^{-1}(\mathrm{MMF})$, formato do fruto (FF), espessura da casca em mm (EC), teores de açúcares $\left({ }^{\circ} \mathrm{BRIX}\right)$, firmeza da casca em N (FC), firmeza da polpa em N (FP), coloração da polpa (CP), coloração externa do fruto (CEF) e padrão de listras da casca (PLC) em frutos de híbridos de melancia.

\begin{tabular}{crrrrrrrrrr}
\hline Híbridos & PROD & MMF & FF & EC & BRIX & FC & \multicolumn{1}{c}{ FP } & CP & CEF & PLC \\
\hline 1x2 & $-41,6$ & $-28,7$ & 7,0 & 10,6 & $-25,0$ & 42,0 & 82,3 & 75,0 & $-18,8$ & 13,3 \\
1x3 & $-26,8$ & $-46,1$ & 17,0 & $-2,1$ & $-30,2$ & 40,9 & 154,8 & 87,5 & $-18,8$ & $-46,7$ \\
1x5 & $-31,0$ & $-34,8$ & 6,0 & 5,8 & 0,0 & 20,2 & 17,7 & 8,3 & $-18,8$ & 0,0 \\
1x6 & $-33,4$ & $-36,5$ & 12,0 & $-4,8$ & $-0,9$ & 25,3 & 14,5 & $-16,7$ & $-37,5$ & 0,0 \\
2x4 & $-29,3$ & $-37,4$ & 8,0 & 16,4 & $-14,7$ & 41,2 & 46,8 & 37,5 & $-18,8$ & 0,0 \\
2x5 & $-20,3$ & $-26,1$ & 8,0 & 2,1 & 8,6 & 36,6 & 19,4 & $-4,2$ & $-37,5$ & 0,0 \\
2x6 & $-27,7$ & $-32,2$ & 5,0 & $-1,6$ & $-25,0$ & 27,2 & 32,3 & 37,5 & $-50,0$ & 0,0 \\
3x4 & $-23,6$ & $-28,7$ & 10,0 & 2,1 & $-17,2$ & 34,2 & 100,0 & 37,5 & $-6,3$ & $-33,3$ \\
$3 \times 5$ & $-39,2$ & $-40,9$ & 14,0 & $-5,8$ & $-31,0$ & 43,6 & 114,5 & 100,0 & $-6,3$ & $-33,3$ \\
3x6 & $-25,5$ & $-30,4$ & 6,0 & 6,9 & 2,6 & 38,5 & $-11,3$ & 0,0 & $-46,9$ & 6,7 \\
4x5 & $-40,3$ & $-41,7$ & $-3,0$ & 21,7 & $-9,5$ & 0,0 & 38,7 & 25,0 & $-37,5$ & 0,0 \\
$4 \times 6$ & $-43,6$ & $-44,3$ & 7,0 & 5,8 & $-15,5$ & 12,8 & 0,0 & $-58,3$ & $-37,5$ & 0,0 \\
5x6 & $-33,7$ & $-29,6$ & 3,0 & 21,7 & $-31,0$ & $-5,1$ & 140,3 & 66,7 & $-37,5$ & 0,0 \\
\hline
\end{tabular}

1- Sandia ${ }^{\circledR}$ (Feltrin); 2 - Crimson Sweet $^{\circledR}$ (Hollar); 3 - Crimson Sweet ${ }^{\circledR}$ (Tecnoseed); 4 - Crimson Sweet ${ }^{\circledR}$ (Topseed); 5 - WMX-001G-14-02-5501pl\#08 e 6 - WMX-001G 14-02-55- 01pl\#09. 
Quanto à espessura da casca e coloração da polpa, apresentaram nove cruzamentos com heterose positiva, sendo seis comuns nas duas características. Para sólidos solúveis, de forma geral, os híbridos apresentaram valores significativos para heterose negativa. Houve heterose positiva entre os híbridos Crimson Sweet ${ }^{\circledR}$ (Hollar) x WMX-001G-14-02-55-01pl\#08 e Crimson Sweet $^{\circledR}$ (Tecnoseed) x WMX-001G-14-02-55-01pl\#09 (Tabela 3).

Não houve expressão de heterose em oito híbridos na característica padrão de listras (Tabela 3), sendo positiva em dois híbridos e, em três, negativa. É de se esperar heterose em todos os cruzamentos de genitores de grupos heteróticos distintos, entretanto, nem sempre se obterá heterose, principalmente quando um dos genitores é pouco adaptado, mesmo que haja divergência (Melchinger, 1999).

\section{Conclusões}

Para heterose em relação à média dos pais, o híbrido Crimson Sweet ${ }^{\circledR}$ (Tecnoseed) x WMX-001G-14-02-5501pl\#09 manifestou heterose positiva para a maioria das características, destacando-se os caracteres de melhor interesse comercial como produtividade, massa média de frutos, teores de açúcares e coloração da polpa.

Para teores de açúcares, maior percentual de heterose positiva foi encontrada no híbrido Crimson Sweet $^{\circledR}$ (Hollar) x WMX-001G-14-02-55-01pl\#08. A melhor combinação híbrida para produtividade e firmeza da casca foi obtida nos cruzamentos com as linhagens experimentais WMX-001G-14-02-55-01pl\#08 e WMX-001G-14-02-55-01pl\#09.

Para heterose padrão, não houve efeito positivo para as características produtividade, massa média de frutos e coloração externa dos frutos. Para coloração da polpa, maiores efeitos heteróticos ocorrem nos cruzamentos em que o genitor Crimson Sweet $^{\circledR}$ (Tecnoseed) se encontra, tanto para heterose tradicional como para heterose padrão.

\section{Referências Bibliográficas}

Bahari, M., Rafii, M.Y., Saleh, G.B., Latif, M.A., 2012. Combining ability analysis in complete diallel cross of watermelon (Citrullus lanatus (Thunb.) Matsum. \& Nakai). The Scientific World Journal, 12, 1-6.

Barros, H.B., Sediyama, T., Teixeira, R.C., Fidélis, R.R., Cruz, C.D., Reis, M.S., 2010. Adaptabilidade e estabilidade de genótipos de soja avaliados no estado do Mato Grosso. Revista Ceres, 27, 359-366.

Bernini, C.S., Parteniani, M.E.A.G.Z., 2012. Estimativas de parâmetros de heterose em híbridos de populações $\mathrm{F}_{2}$ milho ${ }^{1}$. Pesquisa Agropecuária Tropical, 42, 56-62.
Bomfim, I.G.A., Cruz, D.O., Freitas, B.M., Aragão, F.A.S., 2013. Polinização em melancia com e sem semente. FortalezaCE, Embrapa Agroindústria Tropical, 54 p. (Circular Técnica 168).

Borém, A., Miranda, G.V., 2009. Melhoramento de plantas, quinta ed. Viçosa-MG, UFV.

Borém, A., Miranda, G.V., 2013. Melhoramento de plantas, sexta ed. Viçosa-MG, UFV.

Bos, I., Caligari, P., 2011. Selection methods in plant breeding, second ed. London, Chapman and Hall.

Cruz, C.D., 2006. Programa Genes: Aplicativo computacional em genética e estatística, Viçosa-MG, UFV.

Cruz, C.D., Regazzi, A.J., 1997. Modelos biométricos aplicados ao melhoramento genético, primeira ed. Viçosa-MG, UFV.

Embrapa - Empresa Brasileira de Pesquisa Agropecuária, 2009. A cultura da melancia. https://ainfo.cnptia.embrapa.br/digital/bitstream/item/11919/2/ 00081320.pdf (acessado 20 de março de 2018).

Falconer, D.S., 1987. Introdução à genética quantitativa, Viçosa-MG, Imprensa Universitária.

Fehr, W.R. 1987. Principles of cultivars developoment, Theory and Technique, New York, Macmillan.

Ferreira, M.A.J.F., 2005. Técnicas de produção de sementes de melancia via polinizações manuais e controladas, em campo e casa-de-vegetação. Brasília-DF, Embrapa Hortaliças, 7 p. (Circular Técnica 40).

Gardner, C.O., Eberhart, S.A., 1966. Analysis and interpretation of the variety cross diallel and related populations. Biometrics, 22, 439-452.

Godoy, A.R., Higuti, A.R.O., Cardoso, A.I.I., 2008. Produção e heterose em cruzamentos entre linhagens de pepino do grupo caipira. Bragantia, 67, 839-844.

Godoy, A.R., Oviedo, V.R.S., Cardoso, A.I.I., 2005. Análise endogâmica de uma população de pepino caipira. Horticultura Brasileira, 23, 785-788.

Gusmini, G., Wehner, T.C., 2004. Heterosis for yield in a watermelon hybrid. Cucurbit Genetcs Cooperative Report, 1, 43-44.

Leão, D.S.S., Peixoto, J.R., Vieira, J.V., Cecilio Filho, A.B., 2008. Produtividade de melancia em diferentes níveis de Adubação química e orgânica. Bioscience Journal, 24, 32-41.

Marame, F., Dessalegne, L., Fininsa, C., Sigvald, R., 2009. Heterosis and heritability in crosses among Asian and Ethiopian parents of hot pepper genotypes. Euphytica, 168, 235-247.

Martins, J.C.P., Aroucha, E.M.M., Medeiros, J.F., Nascimento, I.B., Paula, V.F.S., 2013. Características póscolheita dos frutos de cultivares de melancia, submetidas à aplicação de bioestimulante. Revista Caatinga, 26, 18-24. 
Melchinger, A.E., 1999. Genetic diversity and heterosis, in: Coors, J.G., Pandey, S., (Ed.), The genetics and exploration of heterosis in crops, ASA/CSSA, p. 99-118.

Miranda Filho, J.B., Geraldi, I.O., 1984. An adapted model for the analysis of partial diallel crosses. Revista Brasileira de Genética, 7, 667-688.

Oliveira, L.M.De, Cavalheiro, V.B.D., Moraes, D.M.De, Tilman, M.A.A., Schuch, L.O.B., 2015. Medição do $\mathrm{CO}_{2}$ como método alternativo para a diferenciação do vigor de lotes de sementes de melancia. Ciência Rural, 45, 606-611.

Pfann, A.Z., Faria, M.V., Andrade, A.A., Nascimento, I.R., Faria, C.M.D.R., Bringhentti, R.M., 2009. Capacidade combinatória entre híbridos simples de milho em dialelo circulante. Ciência Rural, 39, 635-641.

Ramos, A.R.P., Dias, R.C.S., Aragão, C.A., 2009. Densidades de plantio na produtividade e qualidade de frutos de melancia. Horticultura Brasileira, 27, 560-564.
Seagro - Secretaria de Agricultura, Pecuária e Abastecimento, 2017. Começa a colheita de melancia no Tocantins. https://seagro.to.gov.br/noticia/2017/6/21/comeca-a-colheitade-melancia-no-tocantins/ (acessado 24 de abril de 2018).

Silva, M.L., Queiróz, M.A., Ferreira, M.A.J.F., Buso, G.S.C., 2006. Caracterização morfológica e molecular de acessos de melancia. Horticultura Brasileira, 24, 405-409.

Souza, F.F., Dias, R.C.S., Queiróz, M.A., 2013. Capacidade de combinação de linhagens avançadas e cultivares comerciais de melancia. Horticultura Brasileira, 31, 595-601.

Tavares, A.T., Vaz, J.C., Coelho, R.S., Lopes, D.A.S.P., Alves, F.Q.G., Nascimento, I.R., 2018. Aptidão agronômica de genótipos de melancia no sul do estado do Tocantins. Agropecuária Científica no Semiárido, 14, 59-64. 